\title{
Foreword from the New JDIQ Editor-in-Chief
}

It is with great pleasure that I take the helm as the new editor-in-chief (EiC) of the ACM fournal of

Data and Information Quality ( $7 D I Q)$. Today's $7 D I Q$ is a highly reputed journal publishing excellent research contributions and exhibiting also special attention to experience papers coming from the many application areas of data and information quality. This achievement has been made possible thanks to the hard work of the previous EiC, Louiqa Raschid, together with the editorial board. This effort has also led to the very recent inclusion of JDIQ into the Emerging Sources Citation Index (ESCI) by Clarivate Analytics (formerly Thomson Reuters). ESCI is a new index created by Clarivate to cover relatively new publications in the Web of Science, so as to both increase their discoverability and enable Clarivate to start tracking citations of these publications. ESCI also serves as a precursor to inclusion in the more prestigious Science Citation Index Expanded (SCIE), which means that $F D I Q$ has now overcome the first major hurdle for eventual inclusion in the Journal Citation Reports (JCR). We observe that, if accepted in SCIE, the journal would receive an Impact Factor, and therefore inclusion in the ESCI is an important milestone for $7 D I Q$.

During the last few years, the importance and relevance of data and information research in diverse application fields, and in the life of every single individual, has been growing at a tremendous pace. Accessing and evaluating the quality of such data and information has become a fundamental issue. Thus, having a journal dealing with multidisciplinary research across a variety of quality topics is critical. My primary goal as EiC is to make $\mathcal{F D I Q ~ " t h e " ~ t o p ~ r e f e r e n c e ~ v e n u e ~ f o r ~ t h e ~ f i e l d . ~}$

My mission is to maintain the rigorous review process introduced by my predecessor, while trying to reduce the average submission-publication time through a strict monitoring process carried on by the associate editors and primarily by the EiC.

Another main objective is to increase the visibility and impact of the journal as well as to attract a larger number of very good research contributions. There are various actions that could be put in place in order to achieve this goal. For example, I already started to enlarge the editorial board by adding new members from diverse geographical, technical, and institutional settings, with the aim of asking them both to help publicize the journal in previously unreached realms, and to provide new ideas and perspectives. Also, I plan to solicit some multidisciplinary special issues covering specific application fields in which data quality is crucial (e.g., pharmacology, health, biology, government, finance). Each special issue will have at least one guest editor from the specific application domain, coupled with a $F D I Q$ editor. Because special issues are an excellent medium to attract submissions, we may also consider having some of them on "core" FDIQ topics to constitute foundational references for the field. At the same time, we always welcome foundational and survey papers that provide a critical assessment of the state of the art on specific quality topics, while highlighting open research challenges. Finally, I count on the help of the associate editors in scouting notable data quality papers presented at top conferences or at specific data and information quality venues and in suggesting the authors to submit extended versions of their work to the journal.

2017 Copyright is held by the owner/author(s).

1936-1955/2017/10-ART8

https://doi.org/10.1145/3143316 
These are my main ideas for $F D I Q$, but I am sure that there are a lot of other potential directions and initiatives that we can follow, and I am open to receive suggestions from all of you. Please e-mail them to catarci@dis.uniroma1.it.

Tiziana Catarci

Editor-in-Chief 